\title{
ATP-STIMULATED ACCUMULATION OF CALCIUM BY CHROMAFFIN GRANULES AND MITOCHONDRIA FROM THE ADRENAL MEDULLA
}

\author{
Hermann R. K. v. Grafenstein * and Eberhard Neumann \\ Max Planck Institute for Biochemistry, \\ 8033 Martinsried/Munich, F. R. Germany
}

Received September 23, 1983

-5 SUMmARY: In the presence of high concentrations of free $\mathrm{Ca}^{2+}$ $\left(10^{-5}-10^{-4}\right.$ M) chromaffin granules and adrenal medullary mitochondria take un large amounts of $\mathrm{Ca}^{2+}$. At low levels of buffered free $\mathrm{Ca}{ }^{+}\left(<10^{-8} \mathrm{M}\right)$, these mitochondria release almost all of their stored calcium, whereas chromaffin granules retain the major part of their calcium pool. In the presence of MgATP and at $<10^{-8} M$ free sa $^{2+}$, the mitochondria do not take up significant amounts of $\mathrm{Ca}^{27}$. Chromaffin granules, however, under the same conditions, show active Mgatp-dependent $\mathrm{Ca}^{24}$ uptake even at nM-concentrations of free $\mathrm{Ca}{ }^{2+}$. The implications of these results for the calcium metabolism of chromaffin cells are discussed.

Secretion of catecholamines from adrenal medullary cells is initiated by influx of calcium from the extracellular space to the cytoplasma. Chromaffin granules, the storage organelles for these hormones, are known to contain high amounts of calcium (1). Chromaffin granules undergoing exocytosis could, therefore, contribute to the net export of calcium which has entered the cell during stimulation (2). T'o perform this function, three prerequisites are necessary: 1) Chromaffin granules should be able to accumulate calcium in the presence of physiological concentrations of free $\mathrm{Ca}^{2+}$. 2) Chromaffin granules should not release their accumulated calcium under resting conditions. This would implicate that, in vivo, another pumping system removes the released calcium from the cytoplasm. 3) The transport system of

\footnotetext{
* To whom correspondence should be addressed.

Present address: King's College London, Department of Physiology

Strand, London WC 2R 2LS
} 
chromaffin granules should have a higher affinity for calcium than any other intracellular organelle.

It has been shown that the transport of calcium across the membrane of chromaffin granules is a carrier-mediated process $(3-5)$, but the energy source for the accumulation of calcium remains obscure. Reports on the effect of ATP are conflicting (3-7). No information is available on the total calcium content in the presence of physiological concentrations of free $\mathrm{Ca}^{2+}$ nor on the affinity of the uptake system of chromaffin granules and other organelles.

In the present communication a new in vitro assay procedure for calcium uptake and release is described, which was used to demonstrate ATP-stimulated high affinity uptake of calcium by chromaffin granules. The uptake system shows all the above postulated properties.

\section{EXPER IMENTA L}

Chemicals: Percoll was obtained from Pharmacia and dialysed against two changes of a solution containing $10 \mathrm{mM}$ EGTA pH 7.4 and two changes of double destilled water to remove endogenous calcium ( $0.1 \mathrm{mM}$ ). All other Chemicals were of the highest purity commercially available and used without further purification.

Preparation of a particle pellet: Minced tissue was prepared as described previously (8). Equal portions (ca. $10 \mathrm{~g}$ ) were taken up in 10 volumes (ca. $100 \mathrm{ml}$ ) of buffers ("SHE-buffers") containing $0.3 \mathrm{mM}$ sucrose, $10 \mathrm{mM} \mathrm{N}-2$-hydroxyethylpiperazine-N '-ethanesulfonic acid (HEPES) and various concentrations of ethylene-bis-aminoethylether $-N, N, N^{\prime}, N^{\prime}$-tetraacetic acid (EGTA) (see figure legends). The $\mathrm{pH}$ was adjusted with $\mathrm{KOH}$ to 7.4. Resuspended pellets of subcellular particles were prepared as described previously (7), except that SHE-buffers were used.

Incubation: Incubations were carried out in duplicate at various levels of free [ $\left.\mathrm{Ca}^{2+}\right]$ in the presence of buffered Percoll solution either containing or lacking MgATP. Final concentrations were: $608(\mathrm{v} / \mathrm{v})$ Percoll, $10 \mathrm{mM}$ HEPES, $0 \ldots 2 \mathrm{mM}$ EGTA, $0.22 \mathrm{mM}$ calcium acetate, 0 or $4 \mathrm{mM} \mathrm{MgATP,} \mathrm{pH}$ 7.4. For further details see figure legends.

Percoll-centrifugation and fractionation of the gradient: Previously described methods were used (7).

Calcium determination: To precipitate protein and Percoll, and to liberate bound calcium, $0.3 \mathrm{ml}$ of each fraction were mixed with $1.2 \mathrm{ml}$ of a solution containing 7.58 (w/v) trichloracetic acid (TCA) and 0.258 (w/v) lanthanum chloride. The precipitate was pelleted with an Eppendorf-centrifuge for $5 \mathrm{~min}$ at maximal speed. The supernatant fluid was used for calcium determination with a Phillips Pye Unicam atomic absorption spectrophotometer. 
The free calcium concentration of the incubation buffer was estimated by multiplying the apparent dissociation equilibrium constant of the CaEGTA-complex at the pH of the incubation medium with the ratio of the total calcium concentration to the total EGTA concentration. This approximation is applicable, since EGTA is calcium-selective and the apparent dissociation constants of the CaEGTA - and CaATP-complex differ by a factor of $10^{-3}$ (11).

Marker assays and protein determination: Percloric acid - soluble $\mathrm{OD}_{280}$ and total catecholamines, determinated fluorimetrically, were used as markers for chromaffin granules (7). Succinic dehydrogenase (9) was used as a marker for mitochondria, acetylcholine esterase (10) as a marker for plasma membranes.

\section{RESULTS}

Assay procedure for calcium uptake: In all experiments incubation was carried out in the presence of Percoll, which was homogeneously mixed with the incubation buffer and the biological sample. The conditions during incubation, i.e. the presence of EGTA and MgATP, were maintained during gradient formation and fractionation. The presence of calcium buffer and MgATP does not impair the complete separation of high density chromaffin granules and mitochondria on gradients of Percoll (see figs. 1,3).

During gradient formation the cell organelles and the calcium taken up by them segregate into peaks, but the calcium left in the incubation medium remains evenly distributed over the fractions of the Percoll gradient. Care was taken to ensure that there was always a sufficient number of fractions (Nr 1...4) containing a negligible amount of biological material in order to estimate the total calcium concentration in the incubation medium. Two criteria indicate that a cell organelle has taken up calcium: 1) Increase of the calcium peak correlating with an organelle peak or shoulder, 2) Decrease of the calcium evenly distributed over the gradient.

Calcium uptake at high $\left[\mathrm{Ca}^{2+}\right]_{\text {free }}$ : Out of a series of 6 pairs of incubations (gradients $\mathrm{Nr} 1 \ldots 12$ ) at various levels of $\left[\mathrm{Ca}^{2+}\right]_{\text {free' }}$ gradients 7 and 8 were incubated at high $\left[\mathrm{Ca}^{2+}\right]_{\text {free }}$. In the absence of MgATP (gradient 7$),\left[\mathrm{Ca}^{2+}\right]_{\mathrm{free}}$ is $80 \mathrm{um}$. Only 

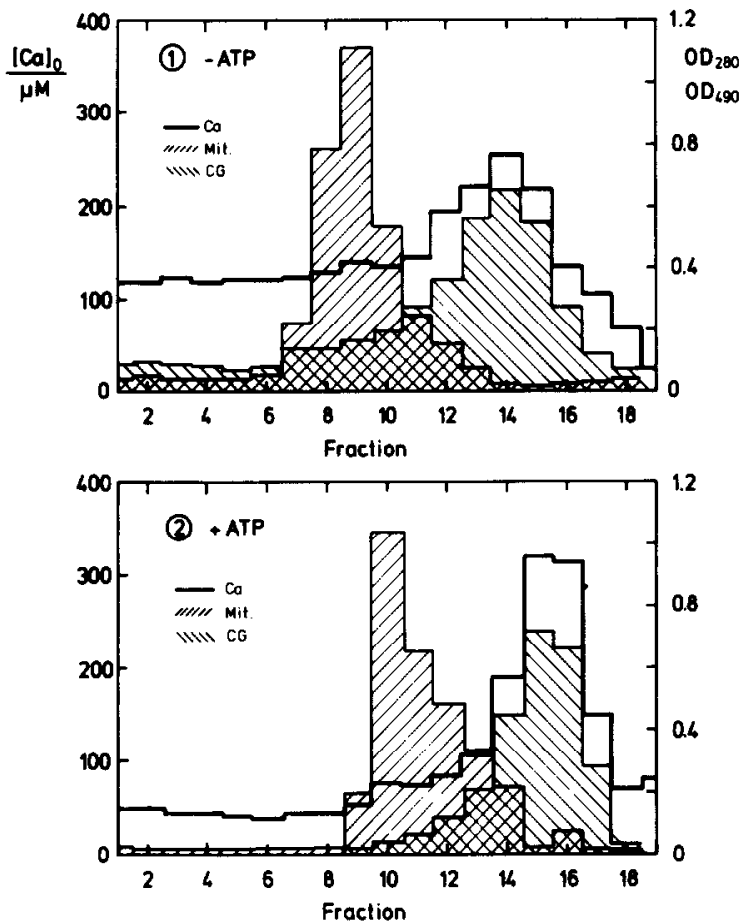

Fig. 1 Calcium uptake at high concentrations of free $\mathrm{Ca}^{2+}$ : The resuspended particle pellet was prepared without EGTA. Equal portions $(0.7 \mathrm{ml})$ were mixed with buffered Percoll either containing MgATP (gradient 8, lower diagram) or lacking MgATP (gradient 7, upper diagram). Both suspensions were incubated for $15 \mathrm{~min}$ at $37^{\circ} \mathrm{C}$ and subsequently transferred to room temperature for gradient-formation and -fractionation. Since both gradients do not contain EGTA, the even part of the calcium profile (fraction 1...4, ) indicates the free calcium concentration after incubation. (Fractions 16-18 cannot be used to estimate free [Ca ${ }^{2+}$, since they are contaminated with sucrose used for pumping out the gradients) Mitochondria: //// Mit, chromaffin granules: $\backslash \backslash \backslash \mathrm{CG}$. The calcium distribution is shown by the bold line (-Ca). The distribution of acetylcholine esterase activity indicates that the observed effects are not due to contamination with plasma membrane fragments (not shown).

chromaffin granules bind calcium. Due to the presence of MgATp (gradient 8$),\left[\mathrm{Ca}^{2+}\right]_{\text {free }}$ is reduced to the detection limit of atomic absorption spectroscopy, which is in the micromolar range under the conditions of these experiments. The shoulder of the calcium profile corresponding with the mitochondrial marker indicates that mitochondria must have accumulated a significant amount of calcium. The major part, however, has been taken up by chromaffin granules (peak height $=480 \mathrm{um}$ ). In this experiment the incubation time at $37^{\circ} \mathrm{C}$ was $15 \mathrm{~min}$. All subsequent procedures were carried out at room temperature, which allows the reaction 

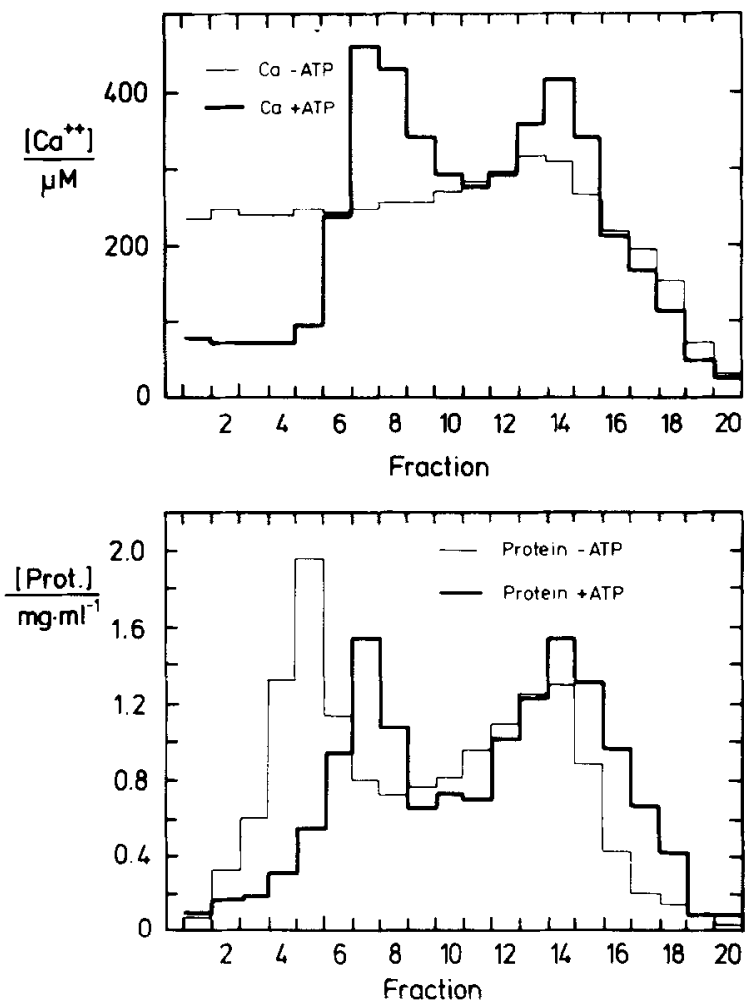

Fig. 2 Calcium uptake at high concentrations of free $\mathrm{Ca}^{2+}$ during short incubation time: The resuspended particle pellet was prepared without EGTA. Equal portions $(0.7 \mathrm{ml})$ were mixed with buffered Percoll either containing MgATP or lacking MgATP. Both suspensions were incubated for $10 \mathrm{~min}$ and subsequently transferred to $0^{\circ} \mathrm{C}$ for gradient formation and fractionation.

Upper diagram: The calcium profile of the MgATP-containing gradient is shown by the bold line (-Ca +ATP), the calcium profile of the MgArp-lacking gradient is shown by a thin line (-..-Ca -ATP). Since both gradients do not contain EGTA, the even part of the calcium profile (fraction 1...3) indicates the free calcium concentration after incubation.

Lower diagram: Protein profile of the ATP-containing gradient (bold line - Protein +ATP) and protein profile of the ATP-lacking gradient (thin line, - Protein -AI'P). The mitochondrial band was centered in fraction-7, the chromaffin granule band was centered in fraction 14.

to continue at a slower rate. If the incubation time is reduced to $10 \mathrm{~min}$, and all subsequent steps are carried out at $0^{\circ} \mathrm{C}$, mitochondria take up more calcium than chromaffin granules (fig. 2). These results suggest that at high levels of $\left[\mathrm{Ca}^{2+}\right]_{\text {free' }}$ both chromaffin granules and mitochondria are abie to accumulate calcium in an ATP-dependent manner. If the free calcium concentration is reduced during prolonged incubation, corresponding to 

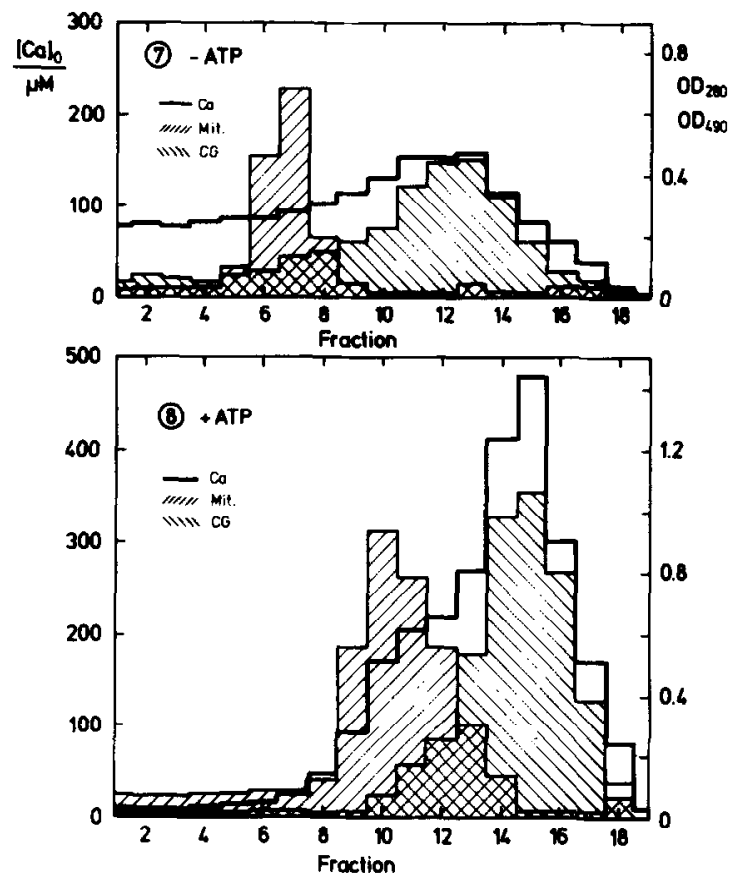

Fig. 3 Calcium uptake at low concentrations of free $\left[\mathrm{Ca}^{2+}\right]$. The resuspended pellet was prepared in the presence of $2 \mathrm{mM}$ EGTA. Subsequent steps see legend to fig.1. Gradient 1 contained $2 \mathrm{mM}$ EGTA, gradient 2 contained $1.8 \mathrm{mM}$ EGTA (due to the addition of ATP-solution). Therefore, the even part of the calcium profile indicates the total calcium concentration in the buffer medium including the CaEGTA-complex and has to be used for the calculation of the free calcium concentration. Mitochondria: //// Mit., chromaffin granules: MMCG. The calcium distribution is shown by the bold line $(-\mathrm{Ca})$.

resting conditions in vivo, mitochondria release their calcium pool which is then accumulated by chromaffin granules.

Calcium uptake at low $\left[\mathrm{Ca}^{2+}\right] \mathrm{free}$ : The results of an experiment carried out at low levels of $\left[\mathrm{Ca}^{2+}\right]$ free are shown in fig. 3 . Due to the presence of MgATP, $\left[\mathrm{Ca}^{2+}\right]_{\text {free }}$ is reduced from $2.2 \mathrm{nM}$ (gradient 1 ) to $0.8 \mathrm{nM}$ (gradient 2 ). In both gradients the calcium profile has only one peak correlating exactly with chromaffin granule markers. This increases due to the presence of MgATP. It can be concluded that at these low levels of $\left[\mathrm{Ca}^{2+}\right]_{\text {free' }}$ mitochondria do not take up calcium. In contrast to mitochondria, chromaffin granules are able to retain a considerable amount of calcium (gradient 1). In the presence of MgATP, they further accumulate calcium and reduce ${ }^{\left[\mathrm{Ca}^{2+}\right.} \mathrm{free}{ }^{\text {. }}$ 


\section{DISCUSS ION}

The results suggest a new model for the feedback regulation of the calcium signal in adrenal medullary cells: Mitochondria act only as a transient calcium buffer and set an upper limit to the cytoplasmic calcium concentration during stimulation. Under resting conditions only chromaffin granules take up and store calcium. They are therefore able to contribute, via exocytosis, to the net export (see also (11)) of calcium which has entered the cell during a previous stimulation period. It is possible that calcium uptake promotes maturation of chromaffin granules from a low to a high density form, which does not take up any more calcium.

ACKNOWLEDGEMENTS: We thank Mr F. Stocker for excelient technical assistence and the Stiftung Volkswagenwerk for financial support.

\section{REFERENCES}

1. Winkler,H. (1977) Neuroscience 2, 657-683

2. Serck-Hanssen,G. \& Christiansen,E.N. (1973), Biochim. Biophys. Acta 587, 75-88

3. Kostron,H., Winkler,H., Geissler,D. \& Konig,P. (1977) $\mathrm{J}$. Neurochem. 28, 487-493

4. Phillips,J.H., (1981) Biochem. J. 200 99-107

5. Krieger-Brauer,H. \& Gratzl, M. (1982) Biochem. Biophys. Acta 691, 61-70

6. Hausler,R., Burger,A. \& Niedermeier,W., (1980) Naunyn-Schmiedeberg's Arch. Pharmacol. 315, 255-267

7. Niedermeier,W. \& Burger, A., (1981) Naunyn-Schmiedeberg's Arch. Pharmacol. 316, 69-80

8. Grafenstein, H.v. \& Neumann, E. (1981) FEBS Lett. 123, 238-240

9. Porteous, J.W. \& Clarc,B. (1967) Biochem. J. 96, 159-171

10. Ellmann,G.L., Courtney,K.D., Andres,V.J. \& Featherstone,R.M. (1961) Biochem. Pharmacol. 7, 88-9l

11. Grafenstein, H.v. Dissertation, Konstañz F.R.G. (1983) 